\title{
Considering Students Seating and Three Effective Classroom Seats Arrangements
}

\author{
IBILOYE Abiodun Christian, ORCiD
}

July 5,2021

\section{Abstract}

Teachers are often challenged on classroom design and space optimization that could bring the best out of learning environment given the available resources. Mostly not serious researches have been published on effects of seats arrangement on cognition, lesson delivery and classroom control. These are part of the contribution made by this article as it review, reflect and offer practical perspectives on three popular student seats arrangements: the traditional long rows (with variants including stadium or lecture theatre design , paired seat rows by columns arrangement, etc.), the U-shape or horseshoe design and the pod-community sitting design. This include principles that educators can consider in choosing arrangements most appropriate for learning and realizing the content and Instructional objectives. Literatures were explored, and from teaching experience, a balance between instructional objectives, class characteristics and diversity, and cost affordable to the school is suggested. Teacher's innovation and improvisation are informed by their exposure and classroom experience. The arrangement of pair desk modules was shown to be the best in all situation, easy to readjust into pod-community design and into u-shape when appropriate for $21^{\text {st }}$ century flipped learning, interactive and collaborative leaning shifts, giving its flexibility, advantage in time before lesson, and with the optimum results.

Keywords: $21^{\text {st }}$ Century Education, Classroom Management, Classroom Seating Arrangement, Instructional Strategies, Pod-Community Sitting, Positive Learning Environment.

\section{Introduction}

Classroom management is the use of teaching techniques, rules and class room space designs and optimization to maximize students' access and response to both teachings and learning materials or supports. That is, teachers must innovatively bring the best out of learning situation, classroom space, and when using technology, and available learning resources. Every classroom is learner-centered by purpose and learning principle, however space characteristics and furniture design impacts on how instructor design instruction and shared materials, how learners sit, receive learning, and they tend to control the chances and form of collaborative and interactive activities, determines teacher' control over learning and instruction process. 
Learning environments symbolize and recreate an institution's vision of educational philosophy, mission commitment and instructional strategies. Traditional seating arrangement does not prohibit collaboration or communication, rather, it makes collaboration, flexible adaptive pedagogy and interactive $21^{\text {st }}$ century skills more challenging. Thus, a class room "designed in a student-centered manner, focusing on learners construction of knowledge and collaboration", as well considering how it helps in maintaining discipline, will really optimize "support [for] student learning." (Rands and Gansemer, 2017).

Academia can be guided by literatures that specifically and sufficiently address tested principles and theories on classroom space management and provide valid statements on the specific effects of seat arrangements on Students cognition support, lesson delivery, time management and classroom control for best instructional results. Though there are journals devoted to learning spaces and researches on flexible seats and sitting arrangements which concentrates survey researches on students and educators self reported preferences and level of satisfactions, very few and no serious empirical studies have examined the direct effects of different seating arrangements on children's cognitive and affective processes, also on potential success of various instructional strategies that could be employed.

Engagement theories like that on school engagements are antiquated and seriously need modern day or $21^{\text {st }}$ century blended application or total update. Brooks, D. Christopher (2012). This article contains informed perspectives, principles, and the context in which school proprietors and educators (in both elementary, secondary schools or within K-12 schools and perhaps higher education) can choose or make innovations/improvisation on three popular seating arrangements: the traditional long rows (with variants including stadium, theatre, or angled row sitting designs), the U-shape or horseshoe design and the team/pod community sitting design.

The aim is to educate and guide educators through modeling and designing classroom sitting arrangement most appropriate for learning and seeing the content and Instructional objectives. Literatures were explored, software assistance suggested for seating plans. From practical teaching experience, instructional efficacy based principle suggest a balance between instructional objectives, class characteristics and diversity and cost affordable to the school. Teacher's innovation and improvisation are informed by their 
exposure and classroom experience, also the school adopted technology and basic Instructional and assessment approaches.

\section{Brief Background and Literature Reviews}

Organization in the classroom is essential, and according to Asino and Pulay (2019), [in Dianala Bernard, (Thursday, 8 July 2021)] “While educational advocates have championed the need for a change to schooling, pedagogy and various teaching and learning practices, ...the physical classroom remains the one constant space where most schooling takes place" (p. 179). Traditional objectives of classroom design and sitting arrangements are to maintain discipline, enable easy movements to sections containing the education materials and allowing the teacher easy and direct contact with the student. Often, we rearrange the learner's seat position either to accommodate a disability or get a trouble some student have the feelings of being observed at all time.

Most elementary classrooms and secondary schools (upper or post-basic schools) offer both the traditional teacher-center classroom arrangements and the contemporary students-centered "open seating" culture, where students self-select their desk and companions on a desk. At times both the class representative and the appointed class teacher do collaborate in select spaces and furniture for newly registered students and teachers can use their discretion to select sitting positions for students that need close proximity to maintain attention or get adequate extra support, often to sit in the front rows.

Activity centers, laboratory, and the type of education event (e.g., examination, lectures or debates), inform a sort of semi-permanent to permanent design of seat arrangement, allocation is as student arrived and choose or still available. Period of occupancy in normal classroom is usually for a session, and given the population size of learners there is no other choice than the traditional arrangement. Teacher and students don't often have ease of movement around. And, in some societies (counties and region of a nation like in Asia, Europe, Australia and West Africa,) religious expectations often influence preference for sectional sitting, usually according to the sex of the students and in extreme small groups of same religion, "socially freezing the class". $21^{\text {st }}$ century skills and technologies are almost impossible to introduce in these contexts aforementioned.

Evidence from the field of Environmental Psychology has "shown that the physical environment of schools and classrooms has an important influence on students' comfort, on their behavior, and on social interactions among peers and between teachers and their students" (Byers, Mahat, Liu, Knock, \& Imms, 2018; Wannarka \& Ruhl, 2008) as stated in 
Tobias, V., et al., (2020). In this paper, three classroom sitting and furniture placement designs are considered. They are briefly accounted for and compared, hence a discussion on the optimum arrangements with a view to suggest guidelines that could be not only insights but great help. From traditional rows and columns to horseshoes and team/pod community setups, there are numerous ways to arrange a classroom; students' sitting position and the seats arrangements. The teacher's decision on whether students can select their own seats, instructional objectives aimed at and the design of the classroom space can have a big influence on equity, educational goals, assessment and discipline in the classroom.

\section{The Popular Three Seating Arrangements}

As part of classroom management strategies, the objectives of sitting arrangement and classroom design include maximizing student learning and having a minimum interactive peer group size, ease of movement, and class control advantages to ensure discipline, orderliness and that a low achiever's learning need is met. As a basic or elementary school teacher, even more the "class advisor/guide" responsible for their registration and attendance registers, you have the unique privilege and task to strategically design and manage physical space of the classroom. This must effectively account for the needs of all students, including those with exceptionalities and behavior nuances.

The three most common models used in classrooms are the traditional long rowand-column model, pairs or pod-community design (as in most elementary schools), and the horseshoe or double horseshoe model.

1. Traditional short columns (pair) and assembly Row Model: Like in all tradional classroom and lecture theatre seating, all the desks are facing the chalkboard/whiteboard and the teacher's desk at a corner or addressing altar at the front center. This layout is particularly very effective if your Instructional Approach is lecture, and your lesson often uses projectors, slides and reading materials. It favors classes with large population sizes i.e., over thirty(30) pupils/students as often seen in developing countries. Student population of fifty to two hundreds (50-200 Students) is often the characteristic of higher institutions' lecture rooms and theatres (particularly social science and arts disciplines) with the chalkboard or whiteboard at the front-center position. Usually noisy!

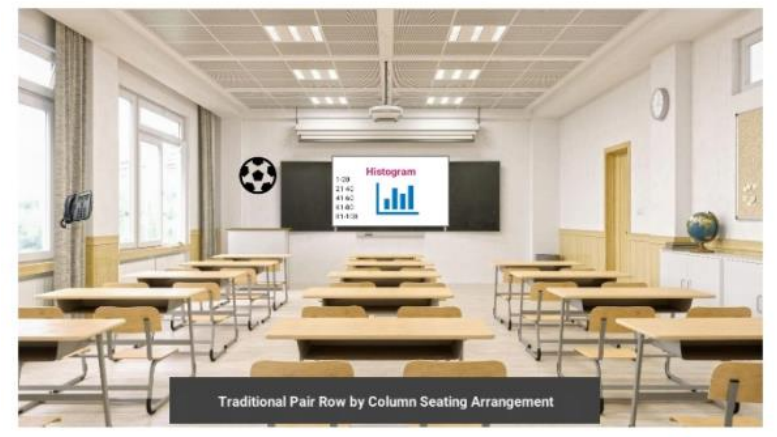


Almost half of the class would end up re-copying notes or photocopying that of those at the front seats. A good lighting system, public address system, emphasis on lecture rules and possibly dividing the class into smaller groups ( $A, B$, and $C$ batches) and increasing the number of instructors could have help maintain standard, Instructional fairness, better chance of education success for many learners and discipline right for positive learning environment.

A variant of this in a classroom is the long desk (three to four student) assembly in columns. Another is a single chair with flip-over book rest/plywood slab for righting notes while students drop their belonging at the sides or at a corner with checkers to keep their belongings. Another arrangement, is a paired (two students per desk with individual or joint seats. With locker system or separate lockers placed at a the corner of the classroom for their bags and over-wears. This allows the teacher to move around the room through the spatial columns.

Whereas, for examination purpose, the arrangement is single person per desk and a good space from next desk which unlike the traditional row-bench design. ensures a fair conduct of the examination, a good optimization of personal space.

\section{Other Advantages}

This is flexible, allows movements of teacher and students, and allows personal choices in learning for the students. Good for higher institutions lecture taking, debates or discussion, secondary schools fixed terminal seating arrangement but unsuitable only for pre-elementary schools and for peer group activities. Cost effective furniture, and rowdy when making major changes.

This is a great classroom seating arrangement for controlled conversations and partner work. Since you've selected the pairs, you can purposefully partner students who may work well together. Also, the interpersonal contact between students due to a low physical distance can positively influence their social perceptions on each other. Wannarka, R., \& Ruhl, K. (2008) believed was "in line with the intergroup contact theory and the contact hypothesis, which state that contact can effectively reduce negative peer perceptions and can increase liking among peers (Allport 1954; Pettigrew 1998). 
However, it is known that large peers grouping relationships ( unlike paired student relationship), are powerful sources of encouraging certain individual behaviors, so students' positive and often negative behaviors in the classroom can be reinforced by peers" (Gest and Rodkin 2011 in ). And, a pupil can easily hide behind peers who are given opportunities to engaged in distractive and gang activities rather than concentrating on learning.

2. Traditional Seating- Long Rows: Usually a design for a lecture, conference, or viewing educational videos where students sit facing the instructor with their backs to one another on long table and benches. This is not suitable, at all, for elementary classrooms, for example, where students takes lessons almost permanently in the room, seating positions fixed for each student and for the term. The teachers generally teach all subject areas, including reading, writing, math, social studies, and science.

If possible, it is helpful to break the rows into column sections, of four pupils but separate chairs. Pupils at the window sides often feel neglected, trapped where it's not easy taking excuses to come out, and most often prefer enjoying the scenes outside the window. While those at the back often becomes irritably noise makers and bunch of gals and guys suffering from Pygmalion effects.

The upper basic or secondary would find this adjusted column design fit for taking lectures and notes despite their large class size. And, I think through the practice of flipped Classroom (a modern teaching method), the students get enough time to

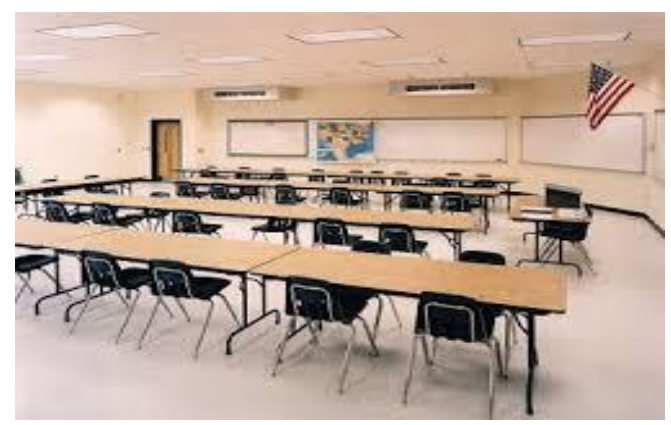
grasp the topic, unlike in a traditional lecture and discussion class where they can only get a few of the materials and notes, as class becomes noisy from the back and the teacher concentrate on those with eye contacts with him/her at the front.

Giving out supplement materials, areas of study and objective points before the class lesson can help prepare these large group of students to respond and make-up for the distance to the teacher by confirming from the given materials, 
hence they can update notes and ask questions even as teacher makes efforts to teach the back of the classroom not to discipline but check on their note taking.

\section{Advantage:}

Students in the front rows have the highest rates of engagement while back rows tend to be less engaged. This style of seating can also be done on an angle, often called stadium seating. In this layout, the desks are in angled rows and may or may not touch, but with good view of and from the teacher. Those with known vision disadvantages can be deliberately relocated to the front seats.

3. U-Shaped or Horseshoe Layout: This fits smaller classes and allows more interaction between the student and educator compared with the other two layouts. A UShaped layout is a better option than stadium design for sciences and demonstration of skill or a process which the learners imitate using their own apparatus and equipment.

This arrangement encourages

discussion and makes it easier than other design for the teacher to observe students and provide one on one help. You'll still have the ability to move freely about the classroom using the indented front u-shaped space. The

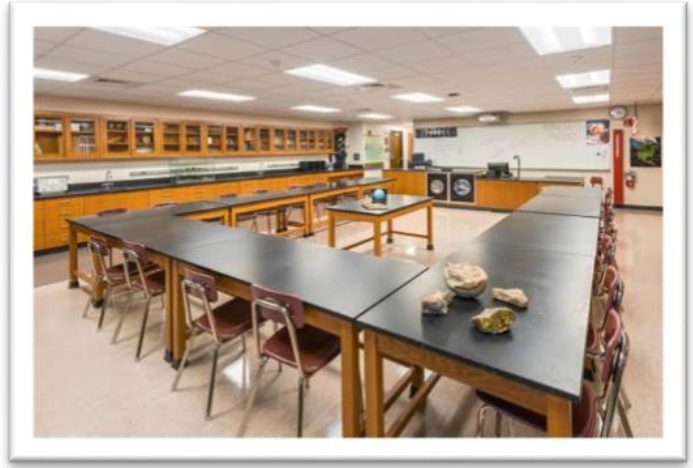
teacher have a strong control on lesson and students response to instruction and discussion, and with side talking discouraged. But, the group dynamic may be lost, especially in classes with a lot of students.

\section{Limitations:}

However, the classroom size, including the teacher- students ratio can determine otherwise, i.e., you may not be able to fit a U-Shape pattern in a small room with a large number of students. The layout spreads children out considerably so that it isn't hard to address them all but it makes group work harder because the desks can't easily be moved around to units for groups. Also, the arrangement of seats hinders small-group discussions because students may often require the full scope of the classroom to communicate across the " $U$ seating design". It makes it easier for some students to cheat during tests because 
their visible range is increased, and multiple angles of view make it practically hard for the teacher to properly police the roaming eyes

4. Pod-Community Sitting: Pod-community seating arrangements involves clustering the students desk into small group (or pod-community seating) which promotes students-to-student interaction, problem solving and motivating leadings on task given to individuals as they watch each other's progress or group collaboration. This option is suitable only for small class size, less than twenty students. And also for syndicate groups in an academic seminar discussion brake-up to sub-units for different or similar task or classes by different instructors.

This team/cluster group offers comfortable environment to share ideas, develop socialacademic bond, critical thinking, respect for diverse opinions and collaboration skills.

Cons: However, it can easily lend itself to off-task activities among children especially in elementary schools, and can increase to noisy level and fights. Group work should be documented for referencing using a seating chart, (Google has a good tool for this). Two students working as a group works great and four students is also a good number, though some students may not work. Groups of three are not recommended because two students will definitely out vote the third and the third student can feel alienated when disagreements arise.

To prevent one student from becoming too dependent on another student, the seating chart can be changed on next task assignment. It's not ideal for conference or lecture where the speaker or instructor must be paid attention in great details. Student cheating and laziness can be remedied by including student participation in the rubric of each assignment. The following example, peer collaboration rubric questions using a rating scale of 1-5, was taken from NGSSlifescience/Classroom Management:

- How much did they work on their own part of the project?

- How much did they help you / partners during class time?

- How useful was their help in the decision making of the project?

- How easy was it to work with the partner?

- How often was he/she on task during class time? 
"Working in groups lets high school, middle school and basic primary or elementary school students rely on each other when they have to do something that is challenging," involving critical thinking, discussion in an English Language or Social Science or History classes.

Technology, like tablet PCs or notebooks can be shared on the joined tables or a table with pair chairs and if in a laboratory, it's perfect for group experiments as they follow the teacher's guidance and leading experiment in steps. While it is hard for every lesson to meet the needs of every student, as a teacher you can meet their needs by incorporating multiple lessons with different modalities.

\section{Setting for Optimum Seating Arrangement.}

Optimizing classroom space, students learning factors and designing the physical components of a classroom are not exactly taught in either teacher's training or coursework but are instincts, pickup skills through examples, practices and logical reflection on classroom characteristics, and also studied behavioral

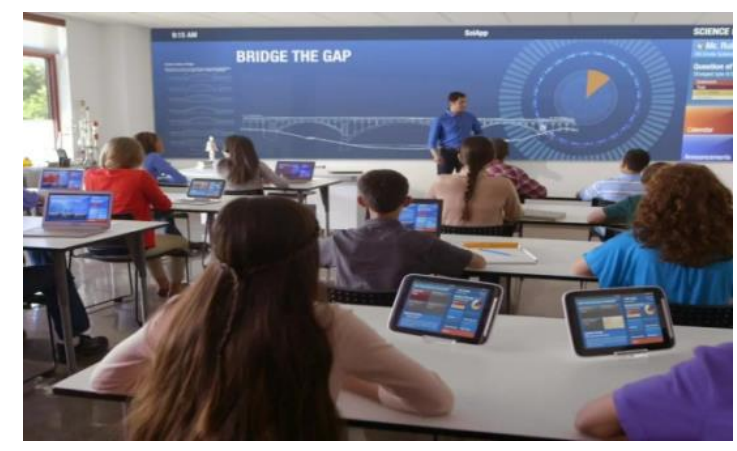
characteristics of the students, which can informed the policy and allocation of sitting position and paired peers.

Teachers or instructor may allow students to choose who they sit next to, which is the most 'student centered approach' which allows students to choose who they collaborate with while clearly showing apathy and prejudice behavior bordering on religion, status or culture, hence not always good for multicultural classroom. There are also different ways to organize the students' sitting positions in your classroom and these include the use of seating charts (which may note student choice and potential social behavior, or alphabetical ordering of their surname and corresponding register/examination numbers).

Some teachers do not believe in using a seating chart, and thus allowing gangs-asfriends to sit next to each other which can cause disruptions in the class. What about substitutes, they may need a seating chart as they do not know the student and their character well enough. Keeping every student engaged in the curriculum, fair access to instruction and help from the teacher, and maintaining discipline are the primary challenge 
for any teacher and should be the ultimate goal. Ideally, your classroom setup should ultimately support movement for students and teacher (no congestion or clutter) and aimed at simple access to materials (dictionaries, textbooks, pencil sharpener, providing solutions on chalkboard if called upon to do so, etc.).

The teacher, therefore, needs to be sensitive to the learning objective of the lesson, as well as the interpersonal dynamic that exists among the students in the group. A good lighting, and clear line of sight, as in either shorter in heights at front or elevated stadium like design, so that you can see the students and they can see you. The trend today is blended learning model, BLM. Hence, in a virtual class meeting the teacher still needs seats charts to be able to manage the virtual class.

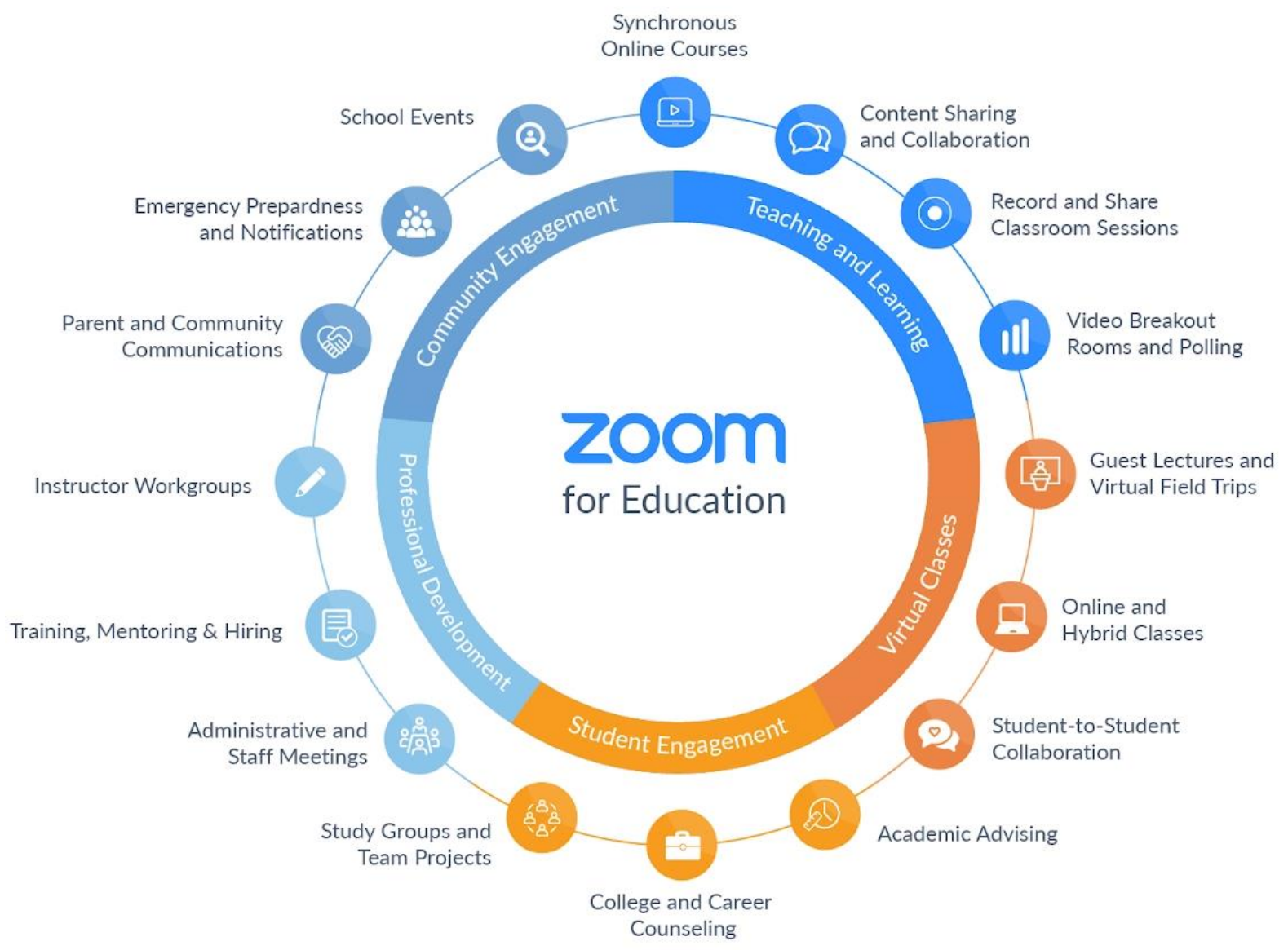

Zoom Virtual Conferencing software has a good collection of features that can help in virtual seating and engagement management.

Today, School Management Software, facilitates most or all of the processes and resources within K-12 schools outside of the classroom. They serve either to centralize schools' administrative (suite) functions or service specific administrative needs or feature solutions. Examples of administrative solutions designed for Education Organization include 
Canvas, Edves Basic and Education Management Solutions, ( which provide complete School Learning \& Management system (Learning, Administration \& Finance) and Blackbaud's Cloud Solutions for K-12 schools and Higher Education and Student Information Management

Learning Management Systems have traditionally been on-premise products [Software-as-a-Product (SaaP) ], that is designed and used only within the organization premises and servers and station system. However, the last 5 years and the normalization of remote learning and work has created a shift of implementation and database to some form of cloud-based standard model for modern LMS, a Software-as-a-Service (SaaS) learning platform. Some vendors offer hybrid deployment approaches, (i.e., Hybrid LMS) which is only necessary in customized cases, such as when there are unusual data privacy or access control requirements. Kathryn Starke, (January 2020)]

Corporate Vendors typically offer either education-focus, management-focus or corporate-staff development Management System. Being a cloud-hosted solution its sometimes affected by poor internet connections in its usage. It's capital intensive and needs constant debugging and software maintenance and upgrade. Sometimes, difficult to customize to real organization structure and function without high cost. Some of these LMS can be integrated with leading third-party video conferencing API such as Zoom, Lessonspace, and Cometchat.

\section{Designing a Digital Seating Charts}

A digital seating charts are just the right way to go in this modern days, and with digital tools like DrawExpress, Google Sheets or Slides or that in Microsoft offices suite, (with thousands of templates you can use or edit). You can begin by measuring your classroom. Then, on Google Slide go to File, Page Setup and choose the ratio that's best for you - or create a custom setup. After the design, the chart with tables bearing students names is added to a 'Google Site' (see the image on the right) or

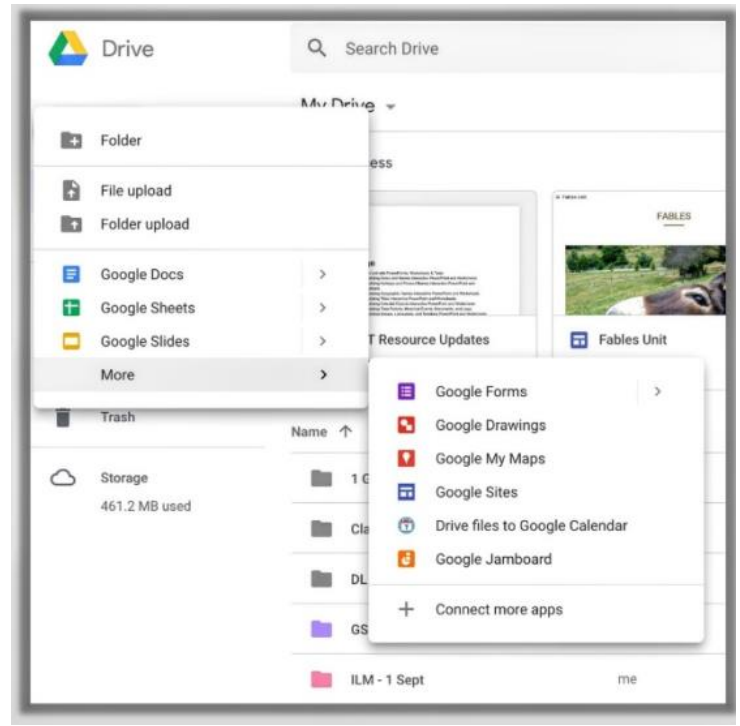
your community website, where you can manage everything with a comprehensive teacher dashboard. From lesson plans to unit plans to long-range plans to sub plans, this website has the power to manage the stuff in your Drive. 


\section{Three Basic Classroom Seating Design Principles}

This section looks at some principle and purpose of the sitting positions. I would like to agree with the statement, "decisions regarding seating arrangements are less difficult when teachers are attuned to a classroom's social dynamics, "which, (increasingly), have been found to be important for positive classroom processes and highly productive convivial peers-teacher environment opined Norwalk et al., 2015. And, based on these principles can alternate the usefulness and appropriateness of the classroom design, especially the sitting arrangements. I here then discuss three composite instructional principles: instructional technique and lesson objectives, communication and conviviality and lastly, discipline and orderliness. Brooks, D. Christopher (2012).

1. Instructional objectives and technique : Although you may not have flexibility over the content of your curriculum, teachers are able to convey information as they see fit on point of lesson delivery. Tailoring your instructional technique to the grade level, subject area, and topic/material objectives is very important. Thus, if you decide on using complementary materials such as video, slides and event type such as seminar, defense or demonstration are best done in stadium like design. Attention is primarily focus on the center of the education, the teacher and the screen or table of apparatus or materials for demonstrating the experiment in a Ushaped arrangements. Researches in the field experimental study suggests the importance of considering both the nature of the task and children's individual characteristics when deciding on a seating arrangement in the classroom. Tobias, V., et al., (2020).

2. Communication and Conviviality: Communication is the most important aspect of classroom management. It is essential to have clear and consistent lines of communication which find support in paired students' resolved needs for assistance from their teacher which could be more complex and difficult for more than two persons comparing notes and synergizing academic strengths. Stadium design cannot give same potential and easy contact and close attention from the teacher but a paired row column arrangements will ensure size good for collaboration and conviviality as they learn and take notes.

Pairing of students is done with the hope that they may benefit from each other, create synergy and grow in productive interaction. Students can build lifelong social- 
academic relationship, distinct world-view, academic culture and self efficacy that will enable them to become increasingly more autonomous, resourceful and less dependent on teacher for mature and informed decisions.

3. Discipline and orderliness: Seating arrangements are important classroom setting and supporting physical resource, because of the potential to help prevent problem of behaviors that decrease student attention and waste available instructional time. Wannarka, R., \& Ruhl, K. (2008) synthesized empirical literature to determine which arrangements of desks best facilitate positive academic and behavioral outcomes for primary through secondary high school students with a range of characteristics. The evidence seems to support "the idea that students display higher levels of appropriate behavior during individual tasks when they are seated in rows, with disruptive students benefiting the most." Wannarka, R., \& Ruhl, K. (2008)

4. Shifts to Interactive, flexible and Collaborative Education. Research studies have shown that collaborative learning environments increase retention by giving students more time to think about, talk about and process information". In addition, collaborative and interactive learning environments promote greater student-toteacher and student-to-student interaction, flexible blended learning, and really support students to be the active in choices towards their education, regaining strength in their weak areas, and facilitate the development of $21^{\text {st }}$ century skills.

Furthermore, the students religion culture and the school preference policy on such culture on are often considered when grouping students for a particular task or practical and in their group seating arrangement. Africans in particular tends to separate the class into sex based sections. This is what is done in Nigeria, and in my school in the northern Muslim predominated region. I can recall same in my secondary school education but partially disappeared in my undergraduate but still we have more often cluster of same sex sitting (of just female or male friends, and just of same religion specifically Muslim women). From my daily experience in teaching, sitting in primary and secondary are fixed, except in laboratory. However, in post-secondary schools, often it's how early you arrive and a good number just stayed at the back with no seat.

Seating preferences of students themselves usually shows the diligent, and more focused students occupy the front rows, in order not to miss out any vital information 
whereas the laid back, more casual students opt for the back benches to avoid being noticed by the educators. Some students even prefer sitting near doors and windows as it provides them with sufficient distractions and outside view to create an escape. You must recognize these behavior and firmly show your preference, and then reallocate or break the class into smaller groups. While, pranks, whispering, passing of notes, quarreling, sleeping, etc. is frequent among students who generally sit in the back, constantly moving towards the place or deliberately involving them in chances to discuss or provide answers can engage them and reduce redundancy related misbehaviors. See also Veenman, S. (1984).

The teachers and particularly the form or class master(adviser) must be conscious of these facts: sometimes, seating arrangements may be a cause for the decline of student performance as attention span, concentration, comprehension and the retaining of information can be influenced by where the student chooses to sit. If the students understand why and how they're expected to use that seating arrangement to their benefit, it can do wonders for engagement.

\section{Conclusion}

It is essential, as educators who desire to help every student as much as possible, to redesign the students seating arrangements to align with our instructional goals and inclusive participation and fair gains in all education activities and instructional contents given the class average age, education technology, the classroom space and method of delivery (discussion, problem solving, peer reflection and discussion activities, demonstration, blended instruction and lecture approach, etc.) so as to maximize student learning, and have advantage at class control- ensuring discipline, participation, assessment and orderliness.

This paper examined, compared and gave informed perspectives on three popular seat arrangements in classroom space design: the traditional arrangements and adjusted model variants, pod-community model, and lastly u-shape design. Digital seating charts are easy to make with Google Slides, Prezi and PowerPoint, and is recommended along with the discussed principles and context of instruction. The goal should be to improve those students' time on task and help individual learning needs through consistent interaction with them, diagnostic assessment, and taking measures for those with learning challenges. Another goal is discipline! "To educate a person in the mind but not in morals is to educate a menace to society." Theodore Roosevelt 
And, "With the current COVID19 situation, it is important to keep all safe health practices, including using facemasks and observing safe distancing. We all have the responsibility to stop the spread of the virus and safety of one another. Google Slides is an easy way to create and track which students are in closer contact in the classroom and good for referencing on need for contact history tracing. It's known some schools have policy that allows religious proprietary. That is, do allow sectioning the classroom into Male and Female sections. I found cooperation the highest in such grouping. However, care must be taken to prevent groups peddling errors copied by just one of them. Classroom seating arrangements affect student learning, motivation, participation, and teacher-student and student-student relationships (Fernandes, Huang \& Rinaldo 2011) .

I have also tried various manipulation of sitting arrangements and re-allocation of space position, the traditional row design with pairs-group desk have not only supported the principles of learning best when it's together (allowing thesis-antithesis discussion on task given) and manageable number to give joint assistance and on-spot assessment as teacher walk through the rows and columns, to inspect, assess and give assistance to paired learners at a time. Two is cooperation, three is a crowd, and if curvilinear rows is choose, then it must be steps as in stadium design, every column a sector shape. This is good in managing small spaces, and for lectures and examination purposes.

Finally, as in the opinion of Dianala Bernard, (Thursday, 8 July 2021), in elementary and basic primary classrooms," for example, where teachers generally teach all subject areas, including reading, writing, math, social studies, and science. If possible, it is helpful to break the room up into sections, for each subject area." With clear rules on their use and protection. A classroom must come alive as a place for discovering, learning and growing academic competence, social and academic communication skills, for applying the acquired knowledge and expressible gifts.

\section{Declaration}

The author declare no other interest than motivation from lectures ( particularly, Unit 4: Using Your Physical Space. M.Ed. Education, (scholarship Programme). University of People, USA) and consequently, my "Research and Practice Portfolio" on Evergreen Notes. The Images are subject to copyright and are from Google drive and search results.. Google Drive/file view, Google Search results 


\section{Cite as:}

Ibiloye, A. C. (2021, July 15)."Considering Students Sitting and Three Effective Classroom Seats Arrangements". Reflection Notes on Educational Research and Practice, EDU 5010. M.Ed Education. University of People. https://doi.org/10.31219/osf.io/7c4qt [APA]

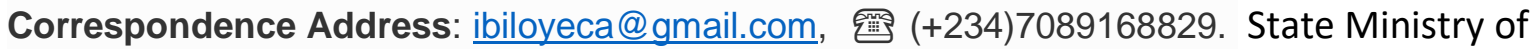
Education Kaduna, Nigeria. Affiliated Institution: Ahmadu Bello University Samaru-Zaria, Nigeria.

Acknowledgement: The author is really grateful for the resources at NGSS Life Science Website, the artifacts from there were really helpful. NGSS Life Science is an online warehouse of lesson plans that were created by our team at NGSS Life Science or teachers all over the United States.

\section{References}

Bloom, L. A. (2009). Classroom management: Creating positive outcomes for all students. Upper Saddle River, NJ: Pearson Education, Inc.

Brooks, D. Christopher (2012). Space and Consequences: The Impact of Different Formal Learning Spaces on Instructor and Student Behavior. Journal of Learning Spaces, 1(2).

Dianala Bernard, (Thursday, 8 July 2021). Using Your Physical Space. Announcement, Unit 4. EDUC 5240-Creating Positive classroom environment. Accessed on July14,2021 at https://my.uopeople.edu/mod/forum/discuss.php?d=558389

Kathryn Starke, (January 13, 2020). "How to Use Learning Management Systems in School. “ https://www.graduateprogram.org/2020/01/how-to-use-learning-management-systems-inschool/ [Kathryn Starke, (January 2020)]

McCroskey, James C.; McVetta, Rod W., (April, 1978). "Classroom Seating Arrangements: Instructional Communication Theory Versus Student Preferences." ED154460

Ming-tak, H., \& Wai-shing, L. (2008). Classroom management: Creating a positive learning environment. Hong Kong University Press. E-book Central, 48-50.

Tobias, V., Sacchi, S., Cerina, V. et al., (2020). The influence of classroom seating arrangement on children's cognitive processes in primary school: the role of individual variables. Curr Psychol. Accessed on July 3, 2021 at https://doi.org/10.1007/s12144-020$\underline{01154-9}$

Veenman, S. (1984). Perceived problems of beginning teachers. Review of Educational Research, 52, 143-178.

Wannarka, R., \& Ruhl, K. (2008). Seating arrangements that promote positive academic and behavioral outcomes: A review of empirical research. Support for Learning, 23, 89-93.

Doi:10.1111/j.1467-9604.2008.00375.x 